\title{
WIND VARIABILITY OF LBV STARS
}

\author{
G. L. ISRAELIAN \\ Byurakan Astrophysical Observatory, Rep. of Armenia
}

\begin{abstract}
General properties of Luminous Blue Variables (LBV) have been reviewed by Lamers(1987). The LBV's are all close to the Humphreys-Davidson luminosity upper limit. The semi periods of the photometric microvariations with $\Delta V \sim 0.1^{\mathrm{m}}$ are about twice as large as for normal supergiants of the same $L$ and $T_{\text {eff }}$, and 4-20 times larger than the fundamental mode for radial pulsations. So, it is likely due to nonradial pulsations. During shell ejections, which accompanied by moderate photometric variations of $\Delta V \sim 1^{m}, L_{\text {bol }}$ remains constant. This fact has been explained by the quasiperiodic variations of $T_{\text {eff }}$ and the radius of pseudo-photosphere (Lamer 1986). LBV's are less stable than normal supergiants so that any internal instability has more effect on their envelopes. The nature of these instabilities remains unknown. We have found some interesting peculiarities which can throw light to this problem.
\end{abstract}

By the comparison of P Cyg CCD spectra obtained by Stahl $\epsilon t$ al. (1990) with that of Johnson et al. (1978), we have found that all 11 comparatively strong emission lines of FeII observed in 1975, disappeared, but lines of FeIII became more intensive. Emission wings of Balmer and some HeI lines also became stronger (Israelian and de Groot 1991a). Obviously, the excitation of FeII atoms for the most part is due to collisions with the free electrons. Lines of [FeII] were stronger in 1975, however, they still exist in 1990 (Stahl et al. 1990). Same kind of things have been observed in stars S Dor (Stahl and Wolf 1982) and R 71 (Wolf et al. 1981), and looks like an expansion of shock waves in the envelopes of long-period variables (Gorbatski 1958). Variations in the ionization degree of metals have been predicted by Pouldrach and Puls (1990), but for a variety of reasons we turn to the shock waves.

A comprehensive analysis of P Cyg CCD spectra permitted to identify 15 from 45 weak hitherto unidentified emission lines (Johnson et al. 1978, Israelian and Nicoghossian 1991). The majority of them belongs to multiionized atoms of metals. (Table I). Therefore an anomalous heating of some layers of the extended envelope can be expected to occur. Some of these unidentified spectral lines exists also in spectra of Sco X-1, $\gamma$ Cas, RR Tel, V 1016 Cyg.

Let us draw a parallel between the evolution of absorption dips (or shells) of $H_{\alpha}$ and propagation of shock waves in the stellar wind of P Cyg. Absorption dips started at $0.4-0.5 \mathrm{~V}_{\mathrm{s}}$ and accelerated with a mean value of $a=0.6$ $\mathrm{cm} / \mathrm{s}^{2}$ until they reach $V=-220 \mathrm{~km} / \mathrm{s}$. This evolution is well approximated with formulae $M=$ const $\rho_{o}^{-1 / \alpha}$ (Castor 1987), where $\alpha=4.9, M$ is Mach value, and $\rho(r)$ accepted from Pouldrach and Puls (1990). It has been shown that if the ratio of the intrinsic line width to the sound speed is large than 
TABLE I

Hitherto Unidentified Emission Lines in P Cyg CCD Spectra (Israelian and Nicoghossian 1991).

\begin{tabular}{llllll}
\hline \multicolumn{2}{c}{ Line } & \multicolumn{2}{c}{ Line } & \multicolumn{2}{c}{ Line } \\
\hline He II & 6890 & {$[\mathrm{Ni} \mathrm{IV}]$} & 5959 & {$[\mathrm{Fe} \mathrm{VII}]$} & 5159 \\
$\mathrm{~N} \mathrm{III}$ & 6465 & {$[\mathrm{Ni} \mathrm{IV}]$} & 5363 & {$[\mathrm{Fe} \mathrm{VII}]$} & 4988 \\
$\mathrm{~N} \mathrm{II}$ & 6173 & {$[\mathrm{Ni} \mathrm{IV}]$} & 5289 & {$[\mathrm{Fe} \mathrm{IV}]$} & 5228 \\
$\mathrm{He} \mathrm{II}$ & 6170 & {$[\mathrm{Ni} \mathrm{IV}]$} & 5040 & {$[\mathrm{Ca}$ VII $]$} & 5619 \\
$\mathrm{Ni}$ I & 5847 & {$[\mathrm{Ca} \mathrm{V}]$} & 5309 & {$[\mathrm{~K} \mathrm{~V}]$} & 4168 \\
\hline
\end{tabular}
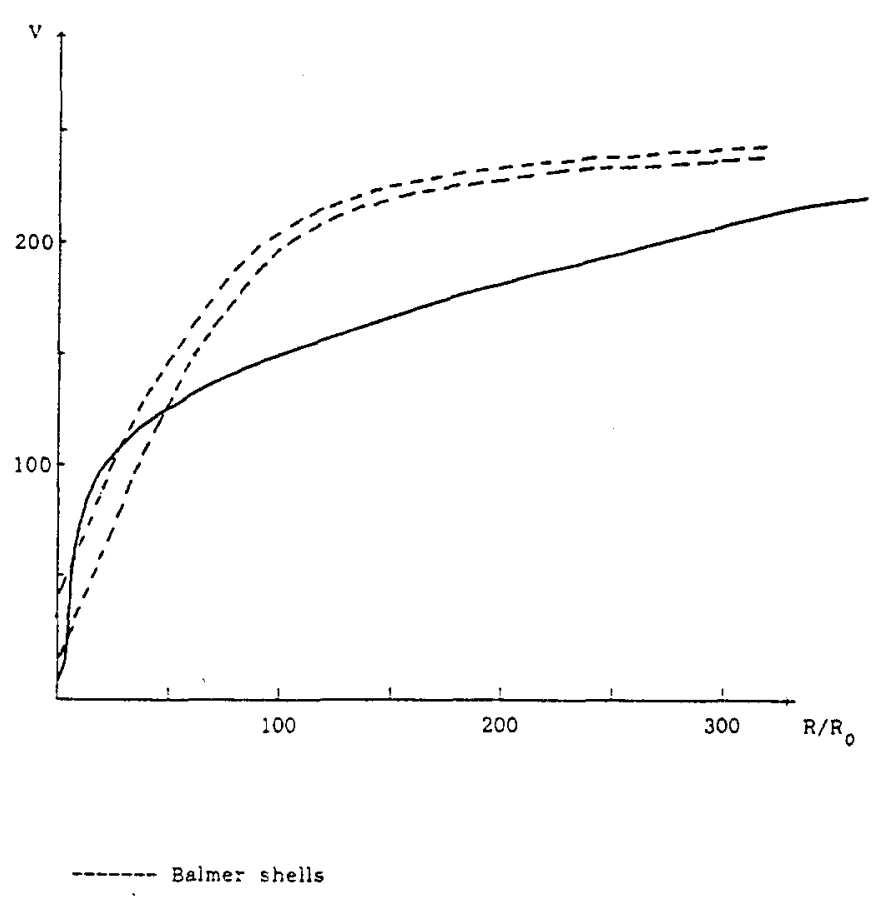

$\longrightarrow$ quiescent wind

Fig. 1.

0.5 , only a negligible wind results; when the ratio is smaller than 0.35 (which is the case of $\mathrm{P} \mathrm{Cyg}$ ), the wind executes permanent self-excited oscillations; in an intermediate range the wind is globally stable, but acts as a powerful wave amplifier (Castor 1990). It is interesting to note that if we compare the velocity law of the Balmer shells (Lamers 1986) with that of the quiescent wind (Pouldrach and Puls 1990, Israeilan and de Groot 1991b), we can see that the accelerations for the shells are at least equal (for smaller $\mathrm{V}$ ) or slightly higher (Fig. 1). It means that these shells are not driven by optically thick lines in the Balmer continuum and there must be another mechanism of the shells acceleration. 

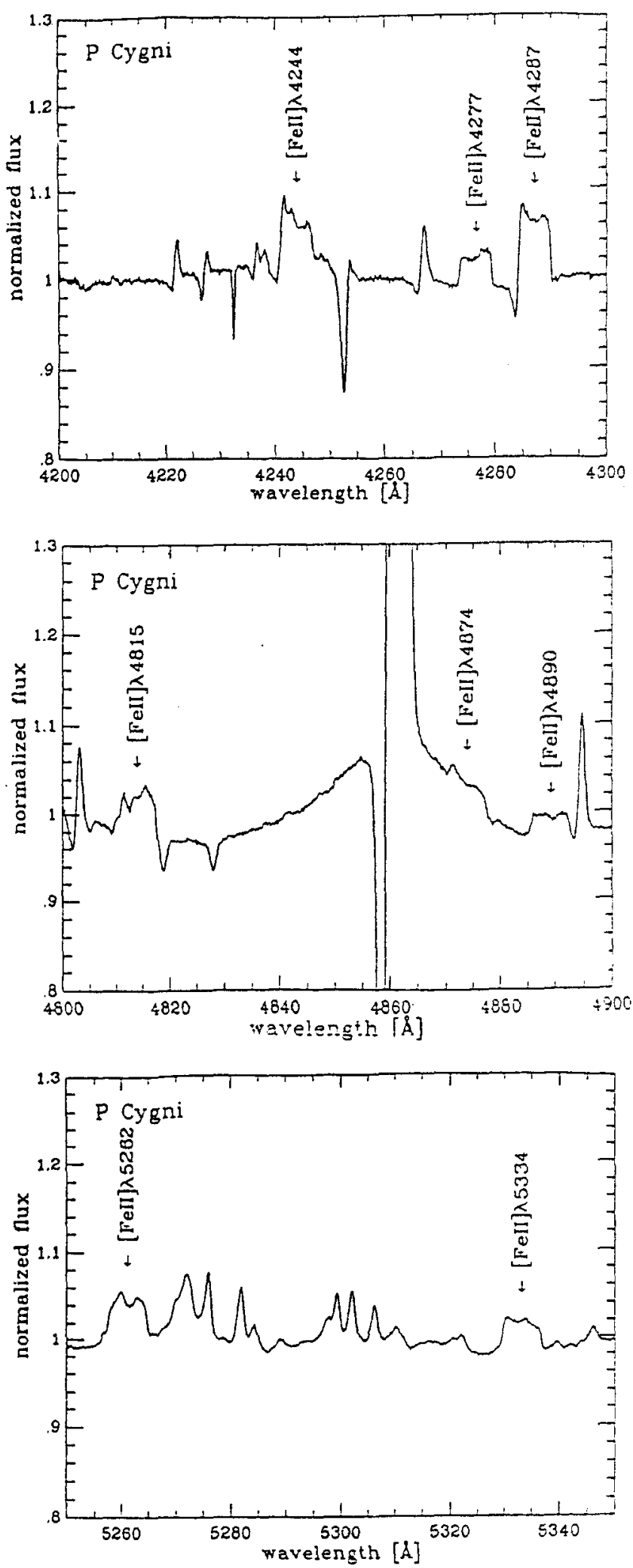

Fig. 2. 
Post-shock radiation can be absorbed in pre-shock region, so the number of free electrons in that region is much more higher and proportional to $V_{s}$. For the optical depth $\tau$ for electron scattering of pre-shock region we have $\tau=14 \mathrm{c} \ln \left(T_{2} / T_{1}\right) / 3 V_{\mathrm{s}}$ (Klimishin 1984) where $T_{2}$ and $T_{1}$ are the post and pre-shock temperatures respectively. In case of adiabatic shock $T_{2} \sim V_{\mathrm{s}}^{2}$ and $T_{2} \gg T_{1}$, but if the post-shock radiation pressure exceeds an adiabatic one, such that $T_{2} \sim V_{s}^{-0.5}$ (Klimishin 1984), then will be $\tau<1$. Expansion of the shock must be accompanied by considerably increasing strengths and extensions of emission line wings. Photometric variations due to electron scattering effects can be expected to occur.

Not long ago Wolf and Stahl (1990) found a change for most of the lines of singly ionized metals from normal to inverse P Cygni type profiles in spectrum of $S$ Dor. We suppose, that this inverse absorption forms in dense post-shock region, as the velocity of this region is less than $V_{s}$ (in an adiabatic case it's equal to $\left.(3 / 4) V_{s}\right)$, and the parcel of material is in free fall between shock passages, as the envelope has a small value of $V_{\text {esc }}$.

At the end, we want to draw attention on emission peaks of [FeII] flattopped profiles (Fig. 2) (Johnson et al. 1978). Obviously these features exist in principle as the CCD spectra has a high ratio $S / N \sim 500$. Much more likely they represent some puffs or blobs of outflowing matter, but there is also the possibility due to thin rotating disk (Underhill and Nemec 1989). Hayes (1985) observed numerous polarization episodes in P Cygni in which $Q$ and $U$ fluctuated irregularly by $0.1-0.5 \%$. These variations were found not to be colinear in $Q$ and $U$ plane, suggesting the existence of anisotropic mass flows in the wind of P Cygni (Underhill and Nemec 1989). This is also supported by last observations held in Byurakan observatory. We have found an increase of $\mathrm{H}$ alpha strength during a 5 days. Another LBV star $\eta \mathrm{Car}$ shows extended jet with the multigraded structure, which also reminds the shock waves (Hayes 1985).

We are grateful to Prof. A. Underhill for helpful discussion and Dr. O. Stahl for an opportunity given by him to analyze his spectra.

\section{References}

Castor, J. I.: 1987, in Instabilities in Early Type Luminous Stars, eds. H. J. G. L. M. Lamers and C. W. H. de Loore (Reidel, Dordrecht), p. 159.

Castor, J. I.: 1990, in NATO Workshop on Stellar Atmospheres, eds. Hubeny and Crivellari (Trieste).

Gorbatski, V. G.: 1958, Soviet Astronomy 35, 5.

Hayes, D. P.: 1985, Astrophysical Journal 258, 639.

Israelian, G. L. and de Groot, M.: 1991a, Astrofis. 34, 3.

Israelian, G. L. and de Groot, M.: 1991b, Astron. Tsirk. 1548, p. 15

Israelian, G. L. and Nicoghossian, A. G.: 1991, Astron. Tsirk., in press .

Johnson, H. L., Wiisniewski, W. Z., and Fay, T. D.: 1978, Rev. Mex. de Astron. Astrof. vol 2 , no 4 , p. 273.

Klimishin, I. A.: 1984, Shock Waves in Stellar Envelopes (Moscow, 'Nayka'), p.75. 
Lamers, H. J. G. L. M.: 1986, Astronomy and Astrophysics 159, 90.

Lamers, H. J. G. L. M.: 1987, in Instabilities in Luminous Early Type Stars, eds. H. J. G. L. M. Lamers and C. W. H. de Loore (Reidel, Dordrecht), p.99.

Pouldrach, A. W. A. and Puls, J.: 1990, Astronomy and Astrophysics 237, 409.

Stahl, O., Mandel, H., Szeifert, Th., Wolf, B. and Zhao, F.: 1990, Astronomy and Astrophysics submitted for publication.

Stahl, O. and Wolf, B.: 1982, Astronomy and Astrophysics 110, 272.

Underhill, A. B. and Nemec, A. F. L.: 1989, Astrophysical Journal 345, 1008.

Wolf, B., Appenzeller, I., and Stahl, O.: 1981, Astronomy and Astrophysics 103, 94.

Wolf, B. and Stahl, O.: 1990, Astronomy and Astrophysics 235, 340. 